\title{
Akustische Therapieverlaufskontrolle nächtlicher Bronchialobstruktionen
}

\author{
U. Koehler \\ V. Gross \\ C. Reinke \\ T. Penzel \\ C. Vogelmeier
}

\author{
Acoustic Follow-up of Nocturnal Bronchial Obstruction Therapy
}

\section{Zusammenfassung}

Langwirksame $\beta_{2}$-Sympathikomimetika gehören zur Basistherapie der COPD. Vor allem Patienten mit nächtlich auftretenden respiratorischen Beschwerden können von dieser Therapie profitieren. Die akustische Langzeitregistrierung der Atemgeräusche ist ein neues Verfahren, das es ermöglicht, bronchiale Obstruktionen im Langzeitverlauf quantitativ zu erfassen. In Kombination mit einer Polysomnographie ist zudem die Beurteilung kardiorespiratorischer Parameter sowie der Schlafstruktur möglich. Bei 10 Patienten (8 Männer und 2 Frauen) mit mittelgradiger COPD $\left(\mathrm{FEV}_{1} 58 \pm 11 \%\right)$ und am Tage auskultatorisch nachweisbarer bronchialer Spastik wurden über zwei bzw. drei Nächte eine akustische Langzeitregistrierung der Atemgeräusche sowie eine Polysomnographie vor und unter erweiterter antiobstruktiver Therapie mit einem langwirksamen inhalativen $\beta_{2}$-Sympathikomimetikum ( $50 \mu \mathrm{g}$ Salmeterol) durchgeführt. Bei allen Patienten konnten nächtliche Bronchialobstruktionen registriert werden. Der Anteil der Wheezing-time am Schlaf fand sich unter Therapie mit Salmeterol in der 1 . Therapienacht mit $33 \pm 17 \%$ vs. $49 \pm 30 \%$ (n.s.) verringert. In der 2 . Therapienacht wurde die Wheezing-time mit $17 \pm 17 \%$ vs. $51 \pm 30 \%(n=6$; $p<0,05)$ bestimmt. Die Schlafeffizienz sowie der prozentuale Anteil an REM-Schlaf waren bei optimierter respiratorischer Situation erhöht (n.s.), der Anteil an Tiefschlaf weitgehend unverändert. Die Reduzierung nächtlicher bronchialer Obstruktionen unter Therapie mit einem langwirksamen $\beta_{2}$-Sympathikomimetikum kann durch das akustische Langzeitmonitoring bestätigt werden. Die Verbesserung der respiratorischen Situation im Schlaf lässt eine Optimierung der Schlafqualität erwarten.

\section{Abstract}

Long acting $\beta_{2}$-agonists belong to the basic therapy of COPD. Especially patients with nocturnal respiratory problems may benefit from this therapy. Long term recording of lung sounds is a new method for quantitative measurements of bronchial obstructions. In combination with polysomnography an evaluation of cardiorespiratory parameters and sleep structure is possible. A total of 10 patients ( 8 male and 2 female) with moderate COPD (FEV1 $58 \pm 11 \%$ ) and signs of bronchial obstruction were investigated. The combination of acoustic long term recording and polysomnography was done for 2 or 3 nights without and under therapy (long acting $\beta_{2}$-agonist, $50 \mu \mathrm{g}$ Salmeterol). In all patients we could find nocturnal bronchial obstruction events. Nocturnal wheezing time was reduced during therapy to $33 \pm 17 \%$ (1. therapy night, n.s.) compared to $49 \pm 30 \%$ without therapy (control night) and to $17 \pm 17 \%$ (2. therapy night, $n=6, p<0.05$ ) vs. $51 \pm$ $30 \%$ (control night, $n=6$ ). Sleep efficiency and REM sleep increased (n.s.) under therapy, deep sleep stages NREM III/IV were nearly the same. Acoustic long term monitoring confirms the reduction of nocturnal bronchial obstructions under therapy with $\beta_{2}$-agonists. A better sleep quality may be expected from the improvement of the respiratory situation during sleep.

Institutsangaben

Klinik für Innere Medizin, SP Pneumologie, Intensiv- und Schlafmedizin, Philipps-Universität Marburg

Danksagung

Wir danken der Fa. Glaxo-Smith-Kline, insbesondere Herrn Dr. Viel,

für die freundliche Unterstützung der Studie.

Korrespondenzadiresse

PD. Dr. med. U. Koehler · Klinik für Innere Medizin · SP Pneumologie, Intensiv- und Schlafmedizin .

Baldingerstraße·35033 Marburg·E-mail: koehleru@mailer.uni-marburg.de

Eingereicht: 3. Oktober 2002 · Nach Überarbeitung angenommen: 30. Januar 2003

Bibliografie

Pneumologie 2003; 57: 198-201 @ Georg Thieme Verlag Stuttgart · New York · ISSN 0934-8387 
Mit der Langzeitregistrierung der Atemgeräusche ist erstmals eine methodische Voraussetzung gegeben, akustische Phänomene wie bronchiale Obstruktionen kontinuierlich über einen längeren Zeitraum zu erfassen [1 -5].

Bronchodilatatoren sind die wichtigsten Medikamente zur symptomatischen Therapie der COPD, hierzu zählen kurz- und langwirksame $\beta_{2}$-Sympathikomimetika, Anticholinergika und Methylxanthine. Die langwirksamen $\beta_{2}$-Sympathikomimetika stellen vor allem bei Patienten mit nächtlich auftretenden respiratorischen Beschwerden eine sinnvolle Therapieoption dar [6-8].

In der vorliegenden Untersuchung wird der Frage nachgegangen, ob der bekannte Effekt der Reduzierung bronchialer Obstruktionen durch eine antiobstruktive Therapie mit einem langwirksamen $\beta_{2}$-Sympathikomimetikum durch ein akustisches Langzeitregistrierverfahren reproduzierbar ist. Gleichzeitig gilt das Interesse der Fragestellung, ob eine Verbesserung der respiratorischen Situation mit einer Optimierung der Schlafqualität einhergeht.

\section{Patienten und Methode}

In die Untersuchung wurden 10 Patienten (8 Männer und 2 Frauen) mit COPD und positivem Auskultationsbefund am Tage (Giemen, Pfeifen und Brummen) aufgenommen. Alle Patienten hatten eine durch Anamnese und Bronchospasmolysetest gesicherte COPD und waren in unterschiedlichster Kombination antiobstruktiv vorbehandelt. 9 von 10 Patienten waren Raucher. Entsprechend den GOLD-Kriterien hatten alle Patienten eine mittelgradige COPD mit einem $\mathrm{FEV}_{1}$ zwischen 30 und $80 \%$ des Sollwertes [9]. Die $\mathrm{FEV}_{1}$-Werte betrugen vor Durchführung unserer Untersuchungen im Mittel $58 \pm 11 \%$ (Bereich: $43-73 \%$ ). Ein Stadium IIB der COPD konnte bei 3 Patienten festgestellt werden.

Das Alter der Patienten lag zwischen 32 und 68 Jahren $(53 \pm 11 \mathrm{~J})$, die Größe zwischen 149 und $192 \mathrm{~cm}(170 \pm 11 \mathrm{~cm})$, der BMI zwischen 19 und $37 \mathrm{~kg} / \mathrm{m}^{2}\left(31 \pm 6 \mathrm{~kg} / \mathrm{m}^{2}\right)$. Bei allen Patienten wurden langwirksame $\beta$-Mimetika mindestens 24 Stunden, kurzwirksame mindestens 8 Stunden vor Durchführung der nächtlichen Registrierungen abgesetzt.

Insgesamt wurden 26 Polysomnographien einschließlich Geräuschaufzeichnung durchgeführt, 4 Untersuchungen in der 3. Messnacht wurden seitens der Patienten abgelehnt, da diese das technisch aufwändige Messequipment der Polysomnographie nicht mehr tolerierten. In der 1. Messnacht erfolgte eine diagnostische Polysomnographie inklusive akustischer Geräuschanalyse. In der 2. und 3. Messnacht wurde den Patienten um 22 Uhr zusätzlich zur bestehenden Medikation $50 \mu$ g Salmeterol (inhalativ) verabreicht.

Für die Aufzeichnung der Lungengeräusche wurde das Gerät PulmoTrack 1010 (Karmel Medical Acoustic Technologies Ltd., Yokneam Illit, Israel) verwendet [2,10]. Dieses Gerät zeichnet kontinuierlich über die gesamte Zeit die Wheezing-Ereignisse mittels 5 piezoelektrischer Sensoren auf. Die Detektion der Auskul- tationsphänomene (Giemen, Pfeifen, Brummen) erfolgt beim PulmoTrack mit einer gewichteten Frequenzanalyse auf der Basis der Fast-Fourier-Transformation. Zur Beschreibung bronchialer Obstruktionen wurden die folgenden Parameter verwendet $[2,10,11]$ :

- Die Wheezing-time-rate (WTR): Die WTR entspricht dem Anteil der erkannten asthmatypischen Nebengeräusche (Brummen, Giemen, Pfeifen) innerhalb von 30-Sekunden-Fenstern.

- Die Wheezing-time (WT): Die WT entspricht der Zeit, für die der Anteil der WTR über 5\% lag (relevante Obstruktion vorhanden).

Bei allen Patienten wurde neben der Aufzeichnung der Lungengeräusche eine Polysomnographie nach Standardkriterien durchgeführt.

Als Maß für eine Veränderung der Parameter unter Therapie wurde der Wilcoxon-Rang-Test berechnet (SPSS 8.0); $\mathrm{p}<0,05$ wurde als signifikanter Unterschied der nicht normalverteilten verbundenen Stichproben angesehen.

\section{Ergebnisse}

Bei allen Patienten konnte eine klinisch relevante schlafbezogene Atmungsstörung ausgeschlossen werden. Der Anteil der Wheezing-time am Schlaf fand sich in der Therapienacht (Nacht 2) mit $33 \pm 17 \%$ vs. $49 \pm 30 \%$ bereits deutlich verringert (n.s.). In der nachfolgenden Therapienacht (Nacht 3), die bei 6 Patienten durchgeführt werden konnte, verringerte sich der Anteil der Wheezing-time (WT) unter Therapie mit Salmeterol signifikant auf $17 \pm 17 \%$ im Vergleich zur diagnostischen Nacht (Nacht 1) mit $51 \pm 30 \%$ WT ( $<<0,05$, Wilcoxon-Test). Abb. 1 demonstriert am Beispiel eines Patienten die Reduzierung bronchialer Obstruktionen unter zusätzlicher antiobstruktiver Therapie mit Salmeterol. Abb. 2 zeigt den Vergleich der prozentualen Anteile der Wheezing-time von diagnostischer Nacht 1 und den Therapienächten 2 und 3. Bei allen Patienten war bei der diagnostischen Polysomnographie eine gestörte Schlafstruktur in Form von mehrfachem Erwachen sowie mangelndem Tief- und REMSchlaf erkennbar. Unter Therapie fanden sich insbesondere der prozentuale Anteil an REM-Schlaf sowie die Schlafeffizienz erhöht (siehe Tab.1).

Eine signifikante Abhängigkeit der Wheezingereignisse von den Schlafstadien konnte bei unseren Patienten nicht nachgewiesen werden, wenngleich eine etwas größere Häufigkeit im REMSchlaf zu verzeichnen war.

\section{Diskussion}

Bei der vorgelegten Arbeit handelt es sich um eine Untersuchung, bei der der therapeutische Effekt eines langwirksamen $\beta_{2}$-Sympathikomimetikums bei Patienten mit mittelgradiger COPD anhand eines Langzeitmonitorings der Atemgeräusche überprüft werden sollte. Bei der durchgeführten Studie ging es weniger um die Bestätigung des bereits bekannten antiobstruktiven Effekts des $\beta_{2}$-Sympathikomimetikums, als um den objektivierbaren Nachweis der Therapieeffizienz durch eine neue di- 
Nacht 1

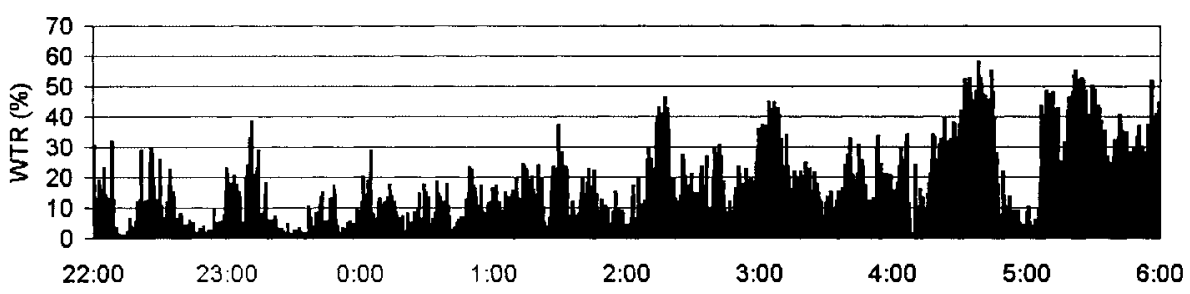

Abb. 1 Gegenüberstellung der nächtlichen Langzeitregistrierung der bronchialen Obstruktionen eines Patienten vor (Nacht 1: diagnostische Nacht) und unter additiver antiobstruktiver Therapie mit einem langwirksamen $\beta_{2}$-Sympathikomimetikum (Nacht 2 und 3).

Nacht 2

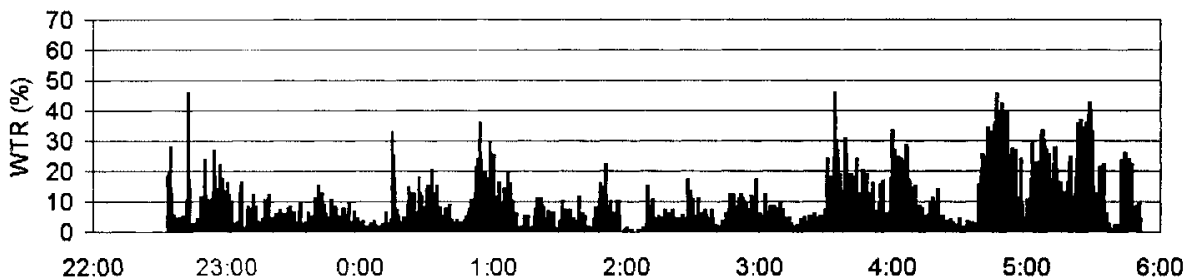

Nacht 3

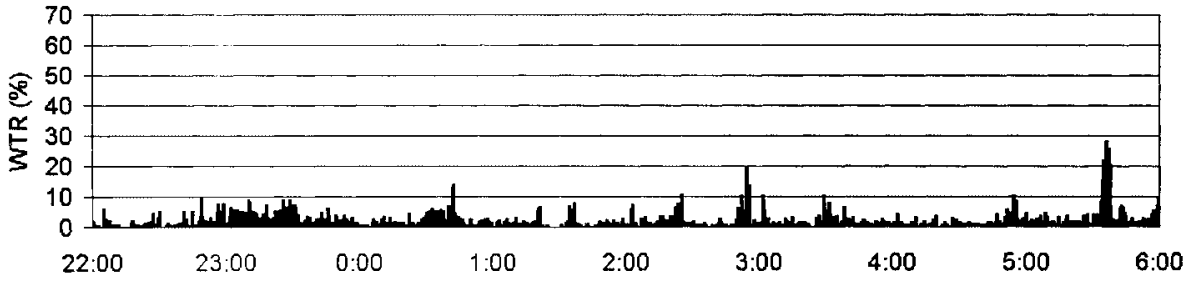

Wheezing time (WT) im Schlaf

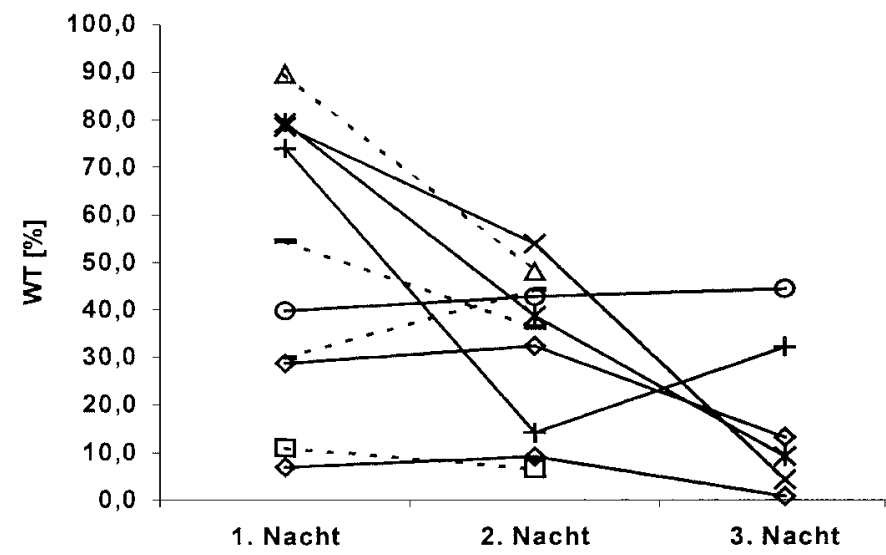

Abb. 2 Wheezing time (WT) der Patienten im Verlauf - 1. Nacht: diagnostische Untersuchung, 2. und 3. Nacht: Untersuchung unter Therapie (durchgehende Linie: 3 Messungen, gestrichelte Linie: 2 Messungen).

agnostische Methode. Um Fremdeffekte durch schlafbezogene Atmungsstörungen sicher auszuschließen, wurde zudem eine Polysomnographie durchgeführt, die gleichzeitig eine Beurteilung der Schlafqualität erlaubt.

Ein wesentlicher Kritikpunkt der Untersuchung ist die Größe des untersuchten Kollektives. Die Problematik ergab sich im Verlauf der Studie dahingehend, dass die Patienten das technisch aufwändige Messequipment der Polysomnographie nur bedingt toleriert haben. So haben 4 der 10 Patienten eine 3. Messnacht verweigert.
Die akustische Lungengeräuschaufzeichnung ist mittlerweile eine anerkannte Methode, deren diagnostische Relevanz anhand mehrerer Studien belegt werden konnte [1-5,10]. Das Langzeitmonitoring der Atemgeräusche stellt ein sinnvolles und effizientes Verfahren zur Beurteilung der antiobstruktiven Therapieeffizienz bei Patienten mit Asthma bronchiale und COPD dar. Unabhängig davon ermöglicht es die frühzeitige Diagnostik von Patienten mit ausschließlich im Schlaf auftretenden respiratorischen Beschwerden im Sinne eines „nächtlichen Asthma bronchiale“. Die synchrone Durchführung von Poly(somno)graphien und Lungengeräuschaufzeichnungen erlaubt zudem die Beurteilung der Interaktion zwischen Schlaf und bronchialer Obstruktion.

Der erweiterte antiobstruktive Therapieeffekt durch ein langwirksames $\beta_{2}$-Sympathikomimetikum konnte bei unseren Patienten durch das Langzeitmonitoring der Atemgeräusche eindrucksvoll bestätigt werden. Die Häufigkeit der in bronchialer Obstruktion verbrachten Zeit war deutlich reduziert. Mit der Verbesserung der respiratorischen Situation war spekulativ auch eine Optimierung der Schlafstruktur erwartet worden. Unsere Untersuchungsergebnisse sind im Hinblick auf die Beurteilung der Schlafqualität aufgrund der geringen Fallzahl jedoch nur sehr eingeschränkt beurteilbar, die erhöhte Schlafeffizienz sowie der erhöhte Anteil an REM-Schlaf lassen jedoch auf eine Verbesserung der Schlafqualität schließen.

Von Patienten mit Asthma bronchiale und COPD ist bekannt, dass sie verstärkt unter Schlafstörungen, Tagesmüdigkeit und einer eingeschränkten Leistungsfähigkeit am Tage leiden [8,12-17]. Dieser Symptomenkomplex ist pathogenetisch durchaus durch eine gestörte Schlafstruktur mit einem Mangel an Tief- und REM- 
Tab. 1 Schlafparameter aller Patienten für den Vergleich 1. und 2. Nacht $(n=10)$ bzw. 1. und 3. Nacht $(n=6)$. Die Verbesserung von Schlafeffizienz, Tiefschlaf- und REM-Anteil sowie Arousal-Index ist statistisch jedoch nicht signifikant.

\begin{tabular}{|c|c|c|c|c|}
\hline & 1. Nacht $(n=10)$ & 2. Nacht $(n=10)$ & 1. Nacht $(n=6)$ & 3. Nacht $(n=6)$ \\
\hline Schlafeffizienz & $64 \pm 23 \%$ & $65 \pm 17 \%$ & $62 \pm 22 \%$ & $70 \pm 14 \%$ \\
\hline Total Sleep Time (TST) & $298 \pm 100 \mathrm{~min}$ & $291 \pm 81 \mathrm{~min}$ & $291 \pm 98 \min$ & $306 \pm 40 \mathrm{~min}$ \\
\hline Schlaflatenz & $65 \pm 81 \mathrm{~min}$ & $42 \pm 24 \mathrm{~min}$ & $49 \pm 37 \min$ & $51 \pm 38 \min$ \\
\hline Anteil NREM $1 / 2$ & $73 \pm 10 \%$ & $67 \pm 8 \%$ & $76 \pm 12 \%$ & $62 \pm 12 \%$ \\
\hline Anteil NREM $3 / 4$ & $14 \pm 7 \%$ & $15 \pm 8 \%$ & $15 \pm 9 \%$ & $16 \pm 7 \%$ \\
\hline Anteil der REM-Phasen & $14 \pm 8 \%$ & $18 \pm 9 \%$ & $10 \pm 6 \%$ & $22 \pm 7 \%$ \\
\hline Resp. Disturbance Index (RDI) & $4,8 \pm 10,4 / h$ & $4,0 \pm 8,1 / h$ & $1,6 \pm 2,1 / h$ & $3,5 \pm 4,4 / h$ \\
\hline Arousal Index (Al) & $51 \pm 22 / h$ & $36 \pm 18 / h$ & $49 \pm 19 / h$ & $30 \pm 12 / h$ \\
\hline
\end{tabular}

Schlaf erklärbar [18]. Es ist demzufolge von großer Relevanz, nachzuweisen, dass es durch einen medikamentös verbesserten respiratorischen Status auch zu einer Verbesserung der Schlafstruktur, insbesondere einer Erhöhung des Tiefschlafanteils kommt.

Langwirksame $\beta_{2}$-Sympathikomimetika stellen einen elementaren Baustein der antiobstruktiven Therapie bei Patienten mit Asthma bronchiale und COPD dar $[6,7,9,19]$. Vor allem Patienten mit im Schlaf auftretenden respiratorischen Beschwerden profitieren von dem Langzeiteffekt dieser Medikation. In unserer Untersuchung wurden ausschließlich Patienten mit mittelgradiger COPD eingeschlossen, die am Tage Zeichen der bronchialen Obstruktion in Form von Giemen, Pfeifen oder Brummen trotz bestehender antiobstruktiver Therapie aufwiesen. Ein solches Patientenkollektiv wurde bewusst gewählt unter dem Aspekt der Beurteilung vorbestehender Geräuschphänomene. Bei Patienten mit chronischer schwergradiger Bronchialobstruktion und einem Lungenemphysem ist die Beurteilung akustischer Phänomene zweifellos eingeschränkt, da diese Patienten eher selten Auskultationsphänomene („silent lung“) aufweisen.

In zukünftigen Untersuchungen wird die Leistungsfähigkeit des Langzeitmonitorings bronchialer Obstruktionen zu überprüfen sein. Es erscheint offensichtlich, dass das Verfahren vor allem bei Patienten mit einem Asthma bronchiale und leichter bis mittelgradiger COPD eine Relevanz haben wird. Möglicherweise ist die Beurteilung von Frequenzspektren der Atemgeräusche aussagefähiger als die ausschließliche Auflistung von Ereignissen wie Giemen, Brummen und Pfeifen.

Um die Frage der pathophysiologischen Verknüpfung von Bronchialobstruktionen und Schlafqualität beantworten zu können, ist die Durchführung von Polysomnographien notwendig. Schlafbezogene Atmungsstörungen, die wiederum selbst Einfluss auf den Schlaf nehmen können, sollten sicher erkannt und von der Bewertung ausgeschlossen werden. Es hat sich gezeigt, dass gerade im Schlaf bedeutsame Interaktionen zwischen kardialem und respiratorischem System bestehen. Vor allem im Rapid-EyeMovement-(REM-) Schlaf kann es zu Prozessabläufen mit einer Desynchronisation von Herz-Kreislauf- und respiratorischen Parametern kommen. Ob der REM-Schlaf, insbesondere für Asthmatiker und COPD-Patienten, ein vulnerables Schlafstadium darstellt, ist eine interessante und klärungsbedürftige Fragestellung.

\section{Literatur}

${ }^{1}$ Baughman RP, Loudon RG. Lung sound analysis for continuous evaluation of airflow obstruction in asthma. Chest 1985; 88 (3): 364-368

2 Kharitonov SA, Kelly C, Godfrey S et al. Continuous, all-night monitoring of breath sounds in stable mild and moderate adult asthmatics on different treatments is feasible and reproducable. Eur Respir J 2000; 16 (31): $534-535$

${ }^{3}$ Koehler U, Gross V, Reinke C et al. Acoustic analysis of nocturnal bronchial obstruction. Pneumologie 2002; 56 (1): 19-24

${ }^{4}$ Pasterkamp H, Wiebicke W, Fenton R. Subjective assessment vs. computer analysis of wheezing in asthma. Chest 1987; 91 (3): 376 - 381

${ }^{5}$ Rietveld S, Oud M, Rijssenbeek-Nouwens LH et al. Characteristics and diagnostic significance of spontaneous wheezing in children with asthma: results of continuous in vivo sound recording. Journal of Asthma 1999; 36 (4): $351-358$

${ }^{6}$ Holimon TD, Chafin CC, Self TH. Nocturnal asthma uncontrolled by inhaled corticosteroids: theophylline or long-acting $\beta_{2}$ agonists? Drugs 2001; 61 (3): $391-418$

${ }^{7}$ Selby C, Engleman HM, Fitzpatrick MF et al. Inhaled salmeterol or oral theophylline in nocturnal asthma? Am J Respir Crit Care Med 1997; 155 (1): $104-108$

${ }^{8}$ Stores G, Ellis AJ, Wiggs L et al. Sleep and psychological disturbance in nocturnal asthma. Arch Dis Child 1998; 78 (5): 413-419

9 Pauwels RA, Buist AS, Calverly PMA et al. Global strategy for the diagnosis, management, and prevention of chronic obstructive pulmonary disease (GOLD). Am J Respir Crit Care Med 2001; 163: 1256-1276

10 Cossa G, Plowman L, MacGarvey L et al. Continuous objective assessment of wheezing in adult asthma patients hospitalised following an acute exacerbation of asthma. Eur Respir J 2001; 18 (33): 267s

${ }^{11}$ Baughman RP, Loudon RG. Quantitation of wheezing in acute asthma. Chest 1984; 86 (5): $718-722$

12 Breslin E, van der Schans C, Breukink S et al. Perception of fatigue and quality of life in patients with COPD. Chest 1998; 114 (4): 958-964

${ }^{13}$ Fitzpatrick MF, Engleman H, Whyte KF et al. Morbidity in nocturnal asthma: sleep quality and daytime cognitive performance. Thorax 1991; 46 (8): $569-573$

${ }^{14}$ Ketelaars CA, Schlosser MA, Mostert R et al. Determinants of healthrelated quality of life in patients with chronic obstructive pulmonary disease. Thorax 1996; 51 (1): 39-43

${ }^{15}$ McSweeny AJ, Grant I, Heaton RK et al. Life quality of patients with chronic obstructive pulmonary disease. Arch Intern Med 1982; 142 (3): $473-478$

16 Okubadejo AA, Jones PW, Wedzicha JA. Quality of life in patients with chronic obstructive pulmonary disease and severe hypoxaemia. Tho$\operatorname{rax} 1996 ; 51$ (1): $44-47$

${ }^{17}$ Schrier AC, Dekker FW, Kaptein AA et al. Quality of life in elderly patients with chronic nonspecific lung disease seen in family practice. Chest 1990; 98 (4): 894-899

${ }^{18}$ Stepanski E, Lamphere J, Badia P et al. Sleep fragmentation and daytime sleepiness. Sleep 1984; 7 (1): $18-26$

${ }^{19}$ Wilson AJ, Gibson PG, Coughlan J. Long acting beta-agonists versus theophylline for maintenance treatment of asthma. Cochrane Database Syst Rev 2000; (2): CD001281 